\section{Knieschmerzen bei Teenies ernst nehmen!}

\begin{abstract}
Wenn Jugendliche über Knieschmerzen klagen, sollte man nicht davon ausgehen, dass diese sich "auswachsen". In einer dänischen Studie waren die Schmerzen bei jedem Zweiten noch nach zwei Jahren vorhanden. Ein Großteil der Betroffenen hatte in diesem Zeitraum sportliche Aktivitäten deutlich eingeschränkt.
\end{abstract}

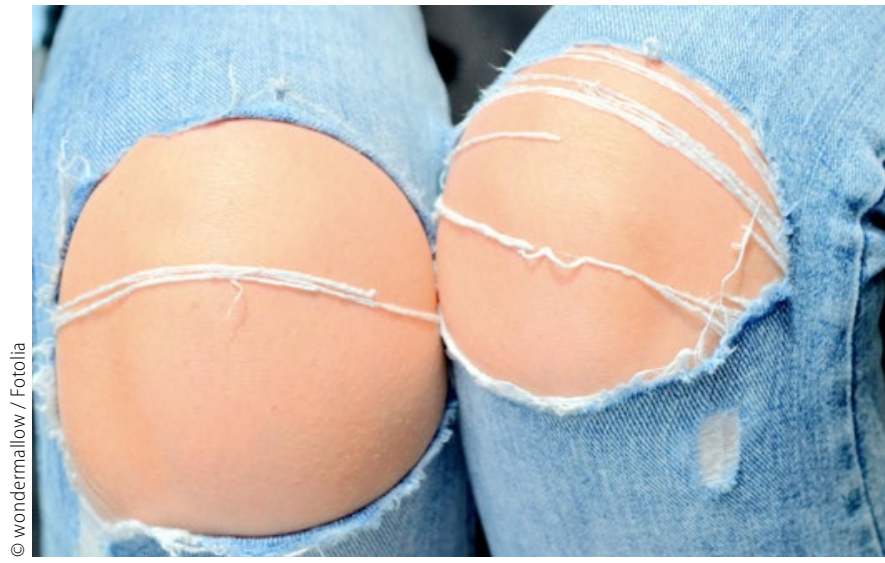

Auch bei Heranwachsenden empfiehlt es sich, Schmerzen im Knie ernst zu nehmen und diese nicht von vornherein als „Wachstumsschmerzen“ abzutun; das rät das Team um Michael S. Rathleff von der Universitätsklinik Aalborg in Dänemark. Die Forscher hatten 504 Patienten im Alter zwischen 15 und 19 Jahren zwei Jahre lang - von 2011 bis 2013 - nachbeobachtet und festgestellt, dass bei $55,9 \%$ die Knieschmerzen danach immer noch vorhanden waren.

Im Vergleich zu primär schmerzfreien Jugendlichen war bei den bereits zu Studienbeginn symptomatischen Teilnehmern die Wahrscheinlichkeit, dass ihnen 2013 das Knie wehtat, um den Faktor 4,51 erhöht. Nach Bereinigung um die Faktoren Alter, Geschlecht und BMI blieb immer noch eine relative Risikoerhöhung von 4,47. Nur 12,8\% der Jugendlichen ohne Knieschmerzen zu Studienbeginn wurde in den darauffolgenden zwei Jahren symptomatisch.

\section{Hartnäckige patellofemorale Schmerzen}

Nach Rathleff und Kollegen waren vor allem patellofemorale Schmerzen (PFS) hartnäckig. Solche hatte man zum Ausgangszeitpunkt bei 153 Patienten diagnostiziert. Das Chronifizierungsrisiko war bei diesem Schmerztyp um 26\% höher als bei Knieschmerzen anderer Lokalisation. Die mittlere Schmerzintensität lag bei den PFS-Patienten auf einer visuellen 100-mm-Analogskala um 13 mm höher als bei Patienten mit anderen Schmerztypen. Im Vergleich zur Gruppe ohne Schmerzen im Jahr 2011 betrug der Unterschied $26 \mathrm{~mm}$. 33\% der Teilnehmer, die 2013 unter PFS litten, hatten täglich Schmerzen, 22\% mehrmals pro Woche. Bei den anderen Schmerztypen lagen die entsprechenden Anteile bei 24 bzw. 13\%.

\section{Jugendliche verzichten auf Sport}

Bedenklich im Hinblick auf die langfristige Entwicklung war, was die Forscher in einer Befragung zu Freizeitaktivitäten fanden: So hatten $71 \%$ der Jugendlichen mit primären PFS nach zwei Jahren ihre sportlichen Aktivitäten deutlich eingeschränkt oder gar komplett gestoppt. Rathleff et al. befürchten, dass der damit erworbene überwiegend sitzende Lebensstil im Erwachsenenalter anhalten und gravierende Folgen für die Herz-Kreislauf-Gesundheit haben könnte.

Vor allem patellofemorale Schmerzen sollte man bei Jugendlichen ernst nehmen, mahnen die Forscher. Charakteristisch für diesen Schmerztyp ist, dass die Beschwerden bei längerem Sitzen, beim Treppensteigen, Hocken oder Knien auftreten und dass die Patella empfindlich auf Berührung reagiert. Entgegen der weit verbreiteten Meinung handelt es sich nach Rathleff et al. sehr oft nicht um selbstlimitierende Wachstumsbeschwerden. Als mögliche Ursachen gelte es, insbesondere Meniskusverletzungen, Tendinopathien, eine Bursitis oder Apophysitis auszuschließen. (Eke Oberhofer)

RathleffMS et al. Is Knee Pain During Adolescence a Self-limiting Condition? Prognosis of Patellofemoral Pain and Other Types of Knee Pain. Am J Sports Med 2016; online 20. Januar; doi: 10.1177/0363546515622456

\section{Karpaltunnelsyndrom}

\section{Kleiner Nutzen durch Elektroakupunktur}

Beim Karpaltunnelsyndrom mäßigen Grades sind konservative Therapieverfahren indiziert. Deren Nutzen ist oft ebenfalls mäßig. Der Erfolg einer Schienung des Handgelenks lässt sich durch eine ergänzende Elektroakupunktur immerhin steigern.

Zum Syndrom gehören klassischerweise auch (nächtlich betonte) Schmerzen. Für chinesische Mediziner um Vincent Chung (Universität Hongkong) eröffnet dies wie selbstverständlich die Frage, ob sich nicht auch beim Karpaltunnelsyndrom (KTS) die Akupunktur segensreich verwenden ließe. In einer Studie mit 181 Teilnehmern haben sie ihre Vermutung überprüft. Diese wurden per Zufall der alleinigen Schienung (91 Probanden) oder der Schienung plus Akupunktur, vorgenommen nach Art der Elektroakupunktur, zugeteilt (90 Probanden).

\section{In nahezu jeder Hinsicht besser}

Abschließend verglichen wurden die Effekte in beiden Gruppen nach 17 Wochen. Tendenziell standen die Probanden der Elektroakupunktur plus SchieneGruppe in nahezu jeder Hinsicht besser da als die Patienten, die nur eine Schiene getragen hatten.

\section{(rb)}

Chung VCH et al. CMAJ 2016, online 6. Juni; doi: $10.1503 / \mathrm{cmaj} .151003$ 